\title{
Influence of dietary fat and carbohydrates proportions on plasma lipids, glucose control and low-grade inflammation in patients with type 2 diabetes-The TOSCA.IT Study
}

\author{
M. Vitale ${ }^{1} \cdot$ M. Masulli ${ }^{1}$ A. A. Rivellese ${ }^{1}$ - A. C. Babini ${ }^{2} \cdot$ M. Boemi $^{3} \cdot$ E. Bonora $^{4}$ ' \\ R. Buzzetti ${ }^{5} \cdot$ O. Ciano $^{1} \cdot$ M. Cignarelli ${ }^{6} \cdot$ M. Cigolini $^{4} \cdot$ G. Clemente $^{1} \cdot$ G. Citro $^{7} \cdot$ L. Corsi $^{8}$. \\ E. Dall'Aglio ${ }^{9}$ S. Del Prato ${ }^{10}$ - G. Di Cianni ${ }^{11} \cdot$ M. A. Dolci ${ }^{12}$ - C. Giordano ${ }^{13}$ - R. Iannarelli ${ }^{14}$. \\ C. Iovine ${ }^{1}$ A. Lapolla ${ }^{15}$ - D. Lauro ${ }^{16}$ - S. Leotta ${ }^{17}$ - C. Mazzucchelli ${ }^{18}$ - V. Montani ${ }^{19}$. \\ G. Perriello $^{20}$ - G. Romano ${ }^{1}$ F. Romeo Re $^{21}$ L. Santarelli2 2 R. Schiano di Cola ${ }^{1}$ - S. Squatrito ${ }^{23}$.

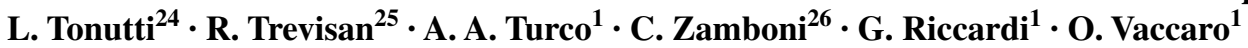

Received: 20 March 2015 / Accepted: 29 June 2015

(C) Springer-Verlag Berlin Heidelberg 2015

\begin{abstract}
Purpose The optimal macronutrient composition of the diet for the management of type 2 diabetes is debated, particularly with regard to the ideal proportion of fat and carbohydrates. The aim of the study was to explore the association of different proportions of fat and carbohydrates
\end{abstract}

Electronic supplementary material The online version of this article (doi:10.1007/s00394-015-0983-1) contains supplementary material, which is available to authorized users.

On behalf of the TOSCA.IT Study Group.

The complete list of Investigators and participating centers is available in the online appendix.

O. Vaccaro

ovaccaro@unina.it

1 Department of Clinical Medicine and Surgery, University "Federico II" of Naples, Naples, Italy

2 Diabetology, Infermi Hospital, Rimini, Italy

3 UOC Malattie Metaboliche e Diabetologia, INRCA-IRCCS Institute, Ancona, Italy

4 Department of Medicine, Division of Endocrinology, Diabetes and Metabolism, University of Verona, Verona, Italy

5 UOC di Diabetologia Universitaria, Ospedale Santa Maria Goretti, Latina, Italy

6 Division of Endocrinology and Metabolism, University of Foggia, Foggia, Italy

7 UO Endocrinologia e Diabetologia, ASP, Potenza, Italy

8 SSD Diabetologia e Malattie del Metabolismo, ASL 4 Chiavarese, Genova, Italy of the diet-within the ranges recommended by different guidelines-with metabolic risk factors.

Methods We studied 1785 people with type 2 diabetes, aged 50-75, enrolled in the TOSCA.IT Study. Dietary habits were assessed using a validated food-frequency questionnaire (EPIC). Anthropometry, fasting lipids, HbA1c and $\mathrm{C}$-reactive protein (CRP) were measured.

Results Increasing fat intake from $<25$ to $\geq 35 \%$ is associated with a significant increase in LDL-cholesterol, triglycerides, $\mathrm{HbA} 1 \mathrm{c}$ and CRP $(p<0.05)$. Increasing carbohydrates intake from $<45$ to $\geq 60 \%$ is associated with significantly lower triglycerides, $\mathrm{HbA} 1 \mathrm{c}$ and

9 Clinical and Experimental Medicine, University of Parma, Parma, Italy

10 Department of Clinical and Experimental Medicine, University of Pisa, Pisa, Italy

11 UOC Diabetologia, ASL 6, Livorno, Italy

12 UO Diabetologia, USL 1, Massa e Carrara, Italy

13 Endocrinology and Metabolic Diseases, University of Palermo, Palermo, Italy

14 UO Diabetologia, Ospedale San Salvatore, L'Aquila, Italy

15 Department of Medicine, University of Padova, Padova, Italy

16 Department of Internal Medicine, Tor Vergata University, Rome, Italy

17 Unit of Diabetology, Sandro Pertini Hospital, Rome, Italy

18 Department of Internal Medicine, University of Genova, IRCCS San Martino, Genova, Italy

19 UOSD, Presidio Ospedaliero di Atri, Atri, Italy

20 MISEM, University of Perugia, Perugia, Italy 
CRP ( $p<0.05)$. A fiber intake $\geq 15 \mathrm{~g} / 1000 \mathrm{kcal}$ is associated with a better plasma lipids profile and lower HbA1c and CRP than lower fiber consumption. A consumption of added sugars of $\geq 10 \%$ of the energy intake is associated with a more adverse plasma lipids profile and higher CRP than lower intake.

Conclusions In people with type 2 diabetes, variations in the proportion of fat and carbohydrates of the diet, within the relatively narrow ranges recommended by different nutritional guidelines, significantly impact on the metabolic profile and markers of low-grade inflammation. The data support the potential for reducing the intake of fat and added sugars, preferring complex, slowly absorbable, carbohydrates.

Keywords Diet - Carbohydrates - Fat - Glucose control . HDL-cholesterol · Triglycerides · Type 2 diabetes .

Nutritional guidelines

\section{Introduction}

The medical nutrition therapy is a cornerstone in the treatment of diabetes; the main goal is to improve glucose control and the cardiovascular risk factors profile [1, 2]. Whereas it is well established that caloric restriction ameliorates glucose control and the plasma lipids profile, mainly through weight loss [3], the optimal macronutrient composition of the diet for the management of patients with type 2 diabetes is still debated. A high protein intake is generally not recommended for patients with diabetes [4]. Therefore, the macronutrient composition of the diet is mainly regulated by the carbohydrate-to-fat ratio, but the ideal proportion of these main food components that should be recommended remains controversial [5-10]. The British Diabetes Association, European Association for the Study of Diabetes (EASD), American Diabetes Association (ADA), American Heart Association, Canadian Diabetes Association, International College of Nutrition, groups from South Africa and Japan, and the National Cholesterol

21 Diabetologia, ASL Torino 5, Torino, Italy

22 Presidio Ospedaliero di Lanciano, Lanciano, Italy

23 Department of Clinical and Experimental Medicine, University of Catania, Catania, Italy

24 SOC di Endocrinologia e Malattie del Metabolismo, AOU "S. Maria della Misericordia", Udine, Italy

25 Endocrinology and Diabetes Unit, AO Papa Giovanni XXIII, Bergamo, Italy

26 Unità Operativa di Malattie Metaboliche, Dietologia e Nutrizione Clinica, AOU Arcispedale "S. Anna", Ferrara, Italy
Education Panel (Adult Treatment Panel 3) have slightly different recommendations for the optimal diet in people with type 2 diabetes [11-16]. The recommended intake of fat ranges from $<25$ to $35 \%$ of total energy intake, whereas the recommended carbohydrates intake varies from 45 to $60 \%$ [17]. Restriction of added sucrose or fructose to a maximum of $10 \%$ of the total energy intake, and fiber intake between 20 and $35 \mathrm{~g} / \mathrm{day}$ are generally recommended. The importance of quality of specific macronutrients and individualized dietary goals is generally emphasized.

One major criticism to these guidelines is that there is insufficient evidence to justify the recommendations. Studies designed to compare the metabolic effects of the various dietary approaches suggested by different organizations for diabetes management are lacking. In particular, the metabolic effects of diets with different proportions of fat and carbohydrates-within the ranges recommended by the nutritional guidelines-have not been explored. Expanding knowledge on this topic would be relevant to help clinicians in the choice of the most suitable dietary intervention(s) to improve the metabolic and cardiovascular risk factors profile in people with diabetes.

The aim of the study is to characterize the habitual diet of a large cohort of people with type 2 diabetes and to explore, in real-life clinical practice, the association of different proportions of fat and carbohydrates of the dietwithin the ranges recommended by different authoritieswith metabolic risk factors. The association of fiber and added sugar with metabolic risk factors is also analyzed.

\section{Materials and methods}

We studied 1785 men and women with type 2 diabetes, aged 50-75 years, enrolled in the TOSCA.IT Study, a randomized clinical trial (clinicaltrials.gov NCT00700856) designed to compare the impact of glucose lowering drugs on cardiovascular event. For the purposes of the present analysis, we used baseline data, collected prior to randomization to study treatment. The study protocol has been approved by the Ethics Review Committee of the coordinating center and of each participating center; written informed consent was obtained from all participants. The details of the study protocol have been published [18]. Body weight, height, waist and hip circumference were measured according to a standard protocol. Body mass index (BMI) was calculated as weight $(\mathrm{kg}) /$ height $\left(\mathrm{m}^{2}\right)$. Biochemical analyses were performed in a central laboratory on fasting samples. Total cholesterol, HDL-cholesterol and triglycerides were measured by standard methods. LDL-cholesterol was calculated according to the Friedewald equation [19] for people with triglycerides values $<400 \mathrm{mg} / \mathrm{dl}$. HbAlc 
Table 1 Clinical characteristics of the study participants and composition of the diet

\begin{tabular}{|c|c|c|}
\hline \multicolumn{3}{|l|}{ Clinical characteristics } \\
\hline$N(\%$ men $)$ & & $(57.7)$ \\
\hline Age (years) & & \pm 6.5 \\
\hline $\mathrm{BMI}\left(\mathrm{Kg} / \mathrm{m}^{2}\right)$ & & \pm 4.4 \\
\hline HbA1c $(\%)$ & & \pm 0.50 \\
\hline HDL-cholesterol (mg/dl) & & \pm 11.9 \\
\hline LDL-cholesterol (mg/dl) & & \pm 30.8 \\
\hline Triglycerides (mg/dl) & & \pm 76.8 \\
\hline Composition of the diet & & Ranges of recommended intake (Ref. [17]) \\
\hline Energy (Kcal/day) & $1898 \pm 673$ & \\
\hline Proteins ( $\%$ of energy) & $18.2 \pm 2.5$ & $10-20$ \\
\hline Fat ( $\%$ of energy) & $36.6 \pm 6.0$ & $25-35$ \\
\hline Saturated fatty acids (\% of energy) & $12.1 \pm 2.5$ & \\
\hline Carbohydrates ( $\%$ of energy) & $45.1 \pm 7.2$ & $45-60$ \\
\hline Fiber (g/day) & $19.4 \pm 2.7$ & $20-35$ \\
\hline Glycaemic index (\%) & $51.7 \pm 3.3$ & \\
\hline Added sugar ( $\%$ of energy) & $2.9 \pm 3.9$ & \\
\hline
\end{tabular}

Data are given as mean \pm standard deviation or proportions

Table 2 Plasma lipids, HbA1c and CRP by intake of fat and carbohydrates (\% of energy intake)

\begin{tabular}{|c|c|c|c|c|c|c|}
\hline \multirow{2}{*}{$\begin{array}{l}\text { Measurements } \\
\text { Total population }\end{array}$} & \multicolumn{3}{|c|}{ Fat ( $\%$ of energy intake) } & \multicolumn{3}{|c|}{ Carbohydrates ( $\%$ of energy intake) } \\
\hline & $<25(n=48)$ & $25-34(n=650)$ & $\geq 35(n=1070)$ & $<45(n=887)$ & $45-59(n=857)$ & $\geq 60(n=31)$ \\
\hline LDL-cholesterol (mg/dl) & $102.0 \pm 3.5$ & $103.3 \pm 3.6$ & $103.7 \pm 3.7 *$ & $103.6 \pm 3.6$ & $103.5 \pm 3.6$ & $103.0 \pm 4.3$ \\
\hline HDL-cholesterol (mg/dl) & $46.1 \pm 3.7$ & $45.5 \pm 3.9$ & $45.5 \pm 3.8$ & $45.5 \pm 3.7$ & $45.5 \pm 3.9$ & $47.0 \pm 3.9$ \\
\hline Triglycerides (mg/dl) & $148.3 \pm 13.6$ & $152.4 \pm 15.2$ & $154.5 \pm 16.0^{*}$ & $154.4 \pm 15.8$ & $153.1 \pm 15.6$ & $144.4 \pm 11.6^{*}$ \\
\hline HbA1c $(\%)$ & $7.67 \pm 0.08$ & $7.70 \pm 0.08$ & $7.71 \pm 0.08 *$ & $7.70 \pm 0.08$ & $7.69 \pm 0.08$ & $7.66 \pm 0.07 *$ \\
\hline $\mathrm{CRP}(\mathrm{mg} / \mathrm{dl})$ & $1.25 \pm 0.11$ & $1.29 \pm 0.13$ & $1.31 \pm 0.14^{*}$ & $1.31 \pm 0.14$ & $1.29 \pm 0.13$ & $1.24 \pm 0.09 *$ \\
\hline$\%$ on lipid-lowering medications & 63.8 & 59.1 & 58.8 & 55.1 & 57.7 & 61.3 \\
\hline
\end{tabular}

Data are given as mean \pm standard deviation or proportions

$* p<0.05$, general linear model (ANCOVA)

was measured with high-performance liquid chromatography (HPLC), and high-sensitivity C-reactive protein (CRP) was measured by Tina-Quant on Roche/Hitachi 904 . At the time of the study, all patients were treated with metformin only: $62 \%$ were on lipid-lowering medications and $90 \%$ were on antihypertensive drugs.

The dietary habits were assessed with the Italian version of the European Prospective Investigation into Cancer and Nutrition (EPIC) questionnaire [20, 21]. The questionnaire contains 248 items including the type of fat used as condiment, or added after cooking. The respondent indicates the absolute frequency of consumption of each item (per day, week, month or year). The quantity of the food consumed was assessed on the basis of the respondent's selection of an image of a food portion showing a small, medium and large portion size, with additional quantifiers (e.g., "smaller than the small portion" or "between the small and medium portion"). The nutrient composition of the diet, the glycemic index and the glycemic load were calculated with the use of a software containing the Italian Food Tables [22, 23] and the International Tables of Glycemic Index [24]. Incomplete questionnaires and questionnaires with implausible data (i.e., energy intake $<800$ or $>5000 \mathrm{kcal} /$ day) were excluded from the analyses.

\section{Statistical analysis}

Data are given as mean and standard deviation or number and proportion, as appropriate. Not normally distributed variables were logarithmically transformed for statistical analyses and back-transformed into natural units for presentation in text and tables. For analytical purposes, 
Table 3 Plasma lipids, HbA1c and CRP by intake of fiber (g/1000 kcal/day) and added sugar (\% of energy intake)

\begin{tabular}{|c|c|c|c|c|c|c|}
\hline \multirow{2}{*}{$\begin{array}{l}\text { Measurements } \\
\text { Total population }\end{array}$} & \multicolumn{3}{|c|}{ Fiber (g/1000 kcal/day) } & \multicolumn{3}{|c|}{ Added sugar (\% of energy intake) } \\
\hline & $<10(n=721)$ & $10-14(n=932)$ & $\geq 15(n=133)$ & $<5(n=1433)$ & $5-9(n=245)$ & $\geq 10(n=90)$ \\
\hline LDL-cholesterol (mg/dl) & $103.5 \pm 3.6$ & $103.4 \pm 3.7$ & $103.8 \pm 3.7$ & $103.4 \pm 3.6$ & $103.7 \pm 3.9$ & $103.9 \pm 4.1$ \\
\hline HDL-cholesterol (mg/dl) & $44.9 \pm 3.8$ & $45.8 \pm 3.7$ & $46.9 \pm 3.7^{*}$ & $45.6 \pm 3.8$ & $45.2 \pm 4.0$ & $45.6 \pm 3.7$ \\
\hline Triglycerides (mg/dl) & $155.0 \pm 15.9$ & $153.1 \pm 15.6$ & $149.3 \pm 14.5^{*}$ & $153.1 \pm 15.5$ & $155.8 \pm 17.1$ & $155.2 \pm 14.8^{*}$ \\
\hline HbA1c $(\%)$ & $7.71 \pm 0.08$ & $7.69 \pm 0.08$ & $7.67 \pm 0.08^{*}$ & $7.69 \pm 0.08$ & $7.71 \pm 0.09$ & $7.72 \pm 0.08 *$ \\
\hline $\mathrm{CRP}(\mathrm{mg} / \mathrm{dl})$ & $1.30 \pm 0.13$ & $1.29 \pm 0.14$ & $1.28 \pm 0.13^{*}$ & $1.30 \pm 0.14$ & $1.32 \pm 0.14$ & $1.33 \pm 0.14^{*}$ \\
\hline$\%$ on lipid-lowering medications & 54.3 & 57.5 & 57.9 & 54.6 & 57.2 & 58.9 \\
\hline
\end{tabular}

Data are given as mean \pm standard deviation or proportions

$* p<0.05$, general linear model (ANCOVA)

Table 4 Plasma lipids, HbA1c and CRP by intake of fat and carbohydrates (\% of energy intake) in the subsample not on lipid-lowering medications

\begin{tabular}{|c|c|c|c|c|c|c|}
\hline \multirow[t]{2}{*}{ Measurements } & \multicolumn{3}{|c|}{ Fat (\% of energy intake) } & \multicolumn{3}{|c|}{ Carbohydrates ( $\%$ of energy intake) } \\
\hline & $<25(n=15)$ & $25-34(n=282)$ & $\geq 35(n=499)$ & $<45(n=419)$ & $45-59(n=365)$ & $\geq 60(n=12)$ \\
\hline LDL-cholesterol (mg/dl) & $101.6 \pm 2.8$ & $103.3 \pm 3.3$ & $103.8 \pm 3.4^{*}$ & $103.7 \pm 3.4$ & $103.5 \pm 3.3$ & $103.2 \pm 4.0$ \\
\hline HDL-cholesterol (mg/dl) & $45.5 \pm 3.7$ & $45.3 \pm 3.7$ & $45.1 \pm 3.6$ & $45.1 \pm 3.6$ & $45.2 \pm 3.8$ & $46.0 \pm 3.7$ \\
\hline Triglycerides (mg/dl) & $148.9 \pm 14.5$ & $153.6 \pm 15.0$ & $156.2 \pm 15.6^{*}$ & $155.5 \pm 15.2$ & $155.0 \pm 15.7$ & $147.6 \pm 13.9 *$ \\
\hline $\mathrm{HbA1c}(\%)$ & $7.67 \pm 0.07$ & $7.70 \pm 0.08$ & $7.71 \pm 0.08 *$ & $7.71 \pm 0.08$ & $7.70 \pm 0.08$ & $7.67 \pm 0.06^{*}$ \\
\hline $\mathrm{CRP}(\mathrm{mg} / \mathrm{dl})$ & $1.25 \pm 0.13$ & $1.29 \pm 0.14$ & $1.31 \pm 0.15^{*}$ & $1.31 \pm 0.14$ & $1.30 \pm 0.14$ & $1.25 \pm 0.12 *$ \\
\hline
\end{tabular}

Data are given as mean \pm standard deviation

$* p<0.05$, general linear model (ANCOVA)

Table 5 Plasma lipids, HbA1c and CRP by intake of fiber (g/1000 kcal/day) and added sugar (\% of energy intake) in the subsample not on lipid-lowering medications

\begin{tabular}{|c|c|c|c|c|c|c|}
\hline \multirow[t]{2}{*}{ Measurements } & \multicolumn{3}{|c|}{ Fiber (g/1000 kcal/day) } & \multicolumn{3}{|c|}{ Added sugar ( $\%$ of energy intake) } \\
\hline & $<10(n=344)$ & $10-14(n=396)$ & $\geq 15(n=56)$ & $<5(n=656)$ & $5-9(n=107)$ & $\geq 10(n=33)$ \\
\hline LDL-cholesterol (mg/dl) & $103.5 \pm 3.3$ & $103.7 \pm 3.4$ & $103.4 \pm 3.4$ & $103.4 \pm 3.3$ & $104.2 \pm 3.6$ & $105.1 \pm 3.4^{*}$ \\
\hline HDL-cholesterol (mg/dl) & $44.5 \pm 3.7$ & $45.5 \pm 3.6$ & $47.0 \pm 3.2^{*}$ & $45.2 \pm 3.7$ & $44.5 \pm 3.7$ & $45.6 \pm 3.7$ \\
\hline Triglycerides (mg/dl) & $157.5 \pm 15.7$ & $154.1 \pm 15.3$ & $147.9 \pm 11.1^{*}$ & $154.3 \pm 15.3$ & $159.1 \pm 15.7$ & $158.1 \pm 15.3^{*}$ \\
\hline $\mathrm{HbA1c}(\%)$ & $7.72 \pm 0.08$ & $7.70 \pm 0.07$ & $7.67 \pm 0.06^{*}$ & $7.70 \pm 0.08$ & $7.73 \pm 0.08$ & $7.72 \pm 0.08 *$ \\
\hline CRP (mg/dl) & $1.31 \pm 0.14$ & $1.30 \pm 0.15$ & $1.28 \pm 0.13 *$ & $1.30 \pm 0.14$ & $1.33 \pm 0.14$ & $1.35 \pm 0.14 *$ \\
\hline
\end{tabular}

Data are given as mean \pm standard deviation

$* p<0.05$, general linear model (ANCOVA)

the dietary intake of the macronutrients of interest was divided into arbitrary categories which reflect the cutoff points derived from different nutritional recommendations (reviewed in [17]), that is, $<25 \%, 25-34 \%$ or $\geq 35 \%$ for total fat intake; $<45 \%, 45-59 \%$ or $\geq 60 \%$ for $\mathrm{CHO} ;<10$, $10-14$ or $\geq 15 \mathrm{~g} / 1000 \mathrm{kcal}$ for fiber and $<5 \%, 5-9 \%$ or $\geq 10 \%$ for added sugar. Plasma lipids, HbAlc and CRP were analyzed across categories of fat, $\mathrm{CHO}$, fiber intake and added sugar by analysis of variance (ANOVA) with post hoc test (Bonferroni). Adjustment for covariates was performed with the general linear model (ANCOVA). The statistical significance was defined as $p<0.05$, two sided. All statistical analyses were performed with the SPSS software for Windows, version 20.0.

\section{Results}

Table 1 gives the clinical characteristics of the study participants along with the composition of the diet and the 
range of intake for proteins, fat, carbohydrates and fiber, recommended by various authorities and summarized in reference [17]. On average, the study participants are overweight/obese, $75 \%$ have abdominal obesity (i.e., waist circumference above $102 \mathrm{~cm}$ for men, or $88 \mathrm{~cm}$ for women) [16]. The glucose control and the plasma lipids profile are close to the treatment targets recommended by the Italian standards of care for people with type 2 diabetes [25]. The energy intake is high $(1898 \pm 673 \mathrm{kcal})$ considering the elevated BMI. The total and saturated fat content of the diet is slightly above recommended. The average carbohydrates content is in the low recommended range $(45.1 \pm 7.2 \%$ of total energy); the glycemic index is low $(51.7 \pm 3.3 \%)$, which suggests that the carbohydrates of the diet are largely represented by slowly absorbable carbohydrates. The fiber intake is lower than recommended ( $19.4 \pm 2.7 \mathrm{~g} /$ day $)$.

As expected, the energy intake is directly associated with plasma triglycerides and inversely associated with HDLcholesterol; no association with LDL-cholesterol, HbA1c and CRP was observed (data not shown). In order to correct for the effect of energy intake in further analyses, the intake of fat, carbohydrates and added sugar is expressed as proportion (\%) of the total energy of the diet and the intake of dietary fiber is expressed as $\mathrm{g} / 1000 \mathrm{kcal} /$ day.

The increase in fat intake from $<25$ to $35 \%$ or more is associated with a significant increase in LDL-cholesterol, triglycerides, HbA1c and CRP ( $p<0.05$ for all) with a significant linear trend (Table 2). To the contrary, increasing carbohydrates intake from $<45$ to $60 \%$ or more is associated with significantly lower triglycerides, $\mathrm{HbA1c}$ and CRP $(p<0.05$ for all), whereas no changes are observed for LDL- or HDL-cholesterol (Table 2).

A consumption of fiber $\geq 15 \mathrm{~g} / 1000 \mathrm{kcal}$ is associated with significantly lower triglycerides, HbAlc and CRP, and higher HDL-cholesterol, compared with a fiber intake $<10 \mathrm{~g} / 1000 \mathrm{kcal}$ (Table 3). To the contrary, plasma triglycerides, HbAlc and CRP increase progressively and significantly with the increasing consumption of added sugar, whereas no effect is observed for LDL- or HDL-cholesterol (Table 3).

The proportion of study participants on lipid-lowering agents is not significantly different across the categories of the nutrients intakes considered (Tables 2,3). A sensitivity analysis, conducted with the exclusion of people on hypolipidemic drugs confirmed the findings (Tables 4, 5).

\section{Discussion}

Although central to medical nutrition therapy, the association of dietary approaches with different proportions of fat and carbohydrates and metabolic risk factors in patients with type 2 diabetes has been little explored. The only meta-analysis which has been performed to quantify the probable metabolic effects of different fat and carbohydrates proportions in people with diabetes was based on intervention studies conducted in small groups of selected patients [5]. Some of the dietary approaches tested included a carbohydrates content as high as $75 \%$ and a fat content as low as $10 \%$ which are out of the recommend ranges for people with diabetes and are also difficult to adhere with in the long term. In addition, studies investigating highcarbohydrates diets also high in dietary fiber were excluded from this analysis [5]. To our knowledge, the present study is the first to characterize the habitual diet of a large cohort of people with type 2 diabetes and to explore, in real-life clinical practice, the association of different proportions of dietary fat and carbohydrates with metabolic risk factors.

The study shows that relatively small variations in the proportion of fat and carbohydrate-within the ranges recommended by most organizations (reviewed in [17]) - are significantly associated with metabolic risk factors and low-grade inflammation, independent of energy intake and use of hypoglycemic drugs. The increase in fat intake from $<25$ to $\geq 35 \%$ is associated with a significant increase in LDL-cholesterol, triglycerides, HbA1c and CRP, whereas the increase in carbohydrates intake from $<45$ to $\geq 60 \%$ is associated with significantly lower triglycerides, $\mathrm{HbA} 1 \mathrm{c}$ and CRP.

The optimal proportion of fat and $\mathrm{CHO}$ of the diet for the management of diabetes is debated [5-10]. Most, though not all, studies show that low-fat/high-carbohydrates diets have a negative impact on plasma triglycerides, HDL-cholesterol, insulin resistance and glucose control [10, 26-28]. The inconsistent findings can be partly explained by differences in the design and analysis of these studies. The lack of standard definitions for high (low)-fat and high (low)-carbohydrates diet makes it difficult to compare different studies. Furthermore, correction for energy intake is not always performed and the source and quality of carbohydrates is rarely taken into account.

In our study population, the main source of carbohydrates is represented by slowly absorbable carbohydrates, as suggested by the low glycemic index of the diet. These characteristics may largely account for the lack of detrimental associations of high carbohydrates consumption with plasma lipids, glucose control and low-grade inflammation. In line with this hypothesis, we find that increasing the intake of fiber is associated with lower triglycerides and CRP and higher HDL-cholesterol levels, whereas the increase in the consumption of added sugars is associated with significantly higher plasma triglycerides, higher CRP and lower HDL-cholesterol. These findings are quite new; whereas the association of dietary fiber with LDL-cholesterol has been extensively investigated, little information 
exists on triglycerides, HDL-cholesterol levels and markers of low-grade inflammation.

Although small in magnitude, the observed metabolic changes are coherent with the results of lifestyle interventions studies [29, 30] and, if translated at the population level, may significantly impact on the population's absolute cardiovascular risk.

The large study population representative of the general diabetic population commonly seen in real-life clinical practice and the central measurement of the metabolic variables (plasma lipids, HbA1c, CRP) are the major study strengths. Among the study limitations, we must acknowledge the observational design and the extensive use of hypolipidemic drugs which, although equally distributed across categories of nutrients intake, may have partly offset the quantitative effect of nutritional factors. However, a sensitivity analysis, performed after exclusion of people on hypolipidemic drugs, confirmed the results, thus strengthening the findings.

In summary, the study shows that in people with type 2 diabetes, changes in the proportion of fat and $\mathrm{CHO}$ of the diet within the relatively narrow ranges proposed by different authorities are associated with significant changes of the metabolic profile and low-grade inflammation The study provides strong observational data in support of the potential for reducing dietary fat intake and added sugars, preferring complex, slowly absorbable, carbohydrates in the habitual diet for patients with type 2 diabetes.

\section{Compliance with Ethical Standards}

Conflict of interest The authors declare that they have no conflict of interest.

Funding The study is supported by the Italian Medicines Agency (AIFA) within the Independent Drug Research Program contract $\mathrm{n}^{\circ}$. FARM6T9CET and by Diabete Ricerca, the no profit Research Foundation of the Italian Diabetes Society.

\section{References}

1. Bantle JP, Wylie-Rosett J, Albright AL, Apovian CM, Clark NG, Franz MJ et al (2008) American Diabetes Association. Nutrition recommendations and interventions for diabetes: a position statement of the American Diabetes Association. Diabetes Care 31(Suppl. 1):S61-S78

2. Ley SH, Hamdy O, Mohan V, Hu FB (2014) Prevention and management of type 2 diabetes: dietary components and nutritional strategies. Lancet 383:1999-2007

3. Heilbronn LK, Noakes M, Clifton PM (1999) Effect of energy restriction, weight loss, and diet composition on plasma lipids and glucose in patients with type 2 diabetes. Diabetes Care 22:889-895

4. Dong JY, Zhang ZL, Wang PY, Qin LQ (2013) Effects of highprotein diets on body weight, glycaemic control, blood lipids and blood pressure in type 2 diabetes: meta-analysis of randomized controlled trials. Br J Nutr 110(5):781-789

5. Kodama S, Saito K, Tanaka S, Maki M, Yachi Y, Sato M et al (2009) Influence of fat and carbohydrate proportions on the metabolic profile in patients with type 2 diabetes: a meta-analysis. Diabetes Care 32:959-965

6. Mann JI, De Leeuw I, Hermansen K, Karamanos B, Karlström B, Katsilambros N, The Diabetes and Nutrition Study Group (DNSG), The European Association for the Study of Diabetes (EASD) et al (2004) Evidence based nutritional approaches to the treatment and prevention of diabetes mellitus. Nutr Metab Cardiovasc Dis 14:373-394

7. Liese AD, Gilliard T, Schulz M, D'Agostino RB Jr, Wolever TM (2007) Carbohydrate nutrition, glycaemic load and plasma lipids: the Insulin Resistance Atherosclerosis Study. Eur Heart J 28:80-87

8. Kirk JK, Craven T, Lipkin EW, Katula J, Pedley C, O'Connor PJ et al (2013) Longitudinal changes in dietary fat intake and associated changes in cardiovascular risk factors in adults with type 2 diabetes: the ACCORD trial. Diabetes Res Clin Pract 100:61-68

9. Kirk JK, Graves DE, Craven TE, Lipkin EW, Austin M, Margolis KL (2008) Restricted-carbohydrate diets in patients with type 2 diabetes: a meta-analysis. J Am Diet Assoc 108(1):91-100

10. Mayer-Davis EJ, Levin S, Marshall JA (1999) Heterogeneity in associations between macronutrient intake and lipoprotein profile in individuals with type 2 diabetes. Diabetes Care 22:1632-1639

11. Nutrition Committee of the British Diabetic Association's Professional Advisory Committee (1992) Dietary recommendations for people with diabetes: an update for the 1990s. Diabet Med 9:189-202

12. National Nutrition Committee CDA (1999) Guidelines for the nutritional management of diabetes mellitus in the new millennium: a position statement by the Canadian Diabetes Association. Can J Diabetes Care 23:56-69

13. Diabetes and Nutrition Study Group, of the European Association for the Study of Diabetes (1995) Recommendations for the nutritional management of patients with diabetes mellitus. Diabetes Nutr Metab 8:186-189

14. American Diabetes Association (2002) Evidence-based nutrition principles and recommendations for the treatment and prevention of diabetes and related complications. Diabetes Care 25:202-212

15. Krauss RM, Eckel RH, Howard BV, Appel LJ, Daniels SR, Deckelbaum RJ, Erdman JW, Kris-Etherton P, Goldberg IJ, Kotchen TA et al (2000) AHA Guidelines Revision 2000: a statement for healthcare professionals from the Nutrition Committee of the American Heart Association. Circulation 102:2284-2299

16. Expert Panel on Detection, Valuation, and Treatment of High Blood Cholesterol in Adults (2001) Executive summary of the third report of the National Cholesterol Education Program (NCEP) Expert Panel on Detection, Evaluation, and Treatment of High Blood Cholesterol in Adults (Adult Treatment Panel III). JAMA 285:2486-2497

17. Ajala O, English P, Pinkney J (2013) Systematic review and meta-analysis of different dietary approaches to the management of type 2 diabetes. Am J Clin Nutr 97:505-516

18. Vaccaro O, Masulli M, Bonora E, Del Prato S, Giorda CB, Maggioni AP et al (2012) Addition of either pioglitazone or a sulfonylurea in type 2 diabetic patients inadequately controlled with metformin alone: impact on cardiovascular events. A randomized controlled trial. Nutr Metab Cardiovasc Dis 22(11):997-1006

19. Fukuyama N, Homma K, Wakana N, Kudo K, Suyama A, Ohazama $\mathrm{H}$ et al (2008) Validation of the Friedewald equation for evaluation of plasma LDL-Cholesterol. J Clin Biochem Nutr 43(1):1-5 
20. Pala V, Sieri S, Palli D, Salvini S, Berrino F, Bellegotti M et al (2003) Diet in the Italian EPIC cohorts: presentation of data and methodological issues. Tumori 89(6):594-607

21. Pisani P, Faggiano F, Krogh V, Palli D, Vineis P, Berrino F (1997) Relative validity and reproducibility of a food frequency dietary questionnaire for use in the Italian EPIC centres. Int J Epidemiol 26(Suppl 1):S152-S160

22. Salvini S, Parpinel M, Gnagnarella P, Maisonneuve P, Turrini A (eds) (1998) Banca dati di composizione degli alimenti per studi epidemiologici in Italia. Istituto Europeo di Oncologia, Milan

23. Carnovale E, Marletta L (eds) (2000) Tabella di composizione degli alimenti. INRAN

24. Foster-Powell K, Holt SHA, Brand-Miller JC (2002) International table of glycemic index and glycemic load values: 2002. Am J Clin Nutr 76:5-56

25. AMD \& SID (2014) Standard Italiani per la cura del diabete mellito. Ed. Infomedica

26. Van Rompay MI, McKeown NM, Castaneda-Sceppa C, Ordovás JM, Tucker KL (2013) Carbohydrate nutrition differs by diabetes status and is associated with dyslipidemia in Boston Puerto Rican adults without diabetes. J Nutr 143(2):182-188
27. Wolever TMS, Nguyen P, Chiason J, Hunt JA, Josse RG, Palmason C et al (1995) Relationship between habitual diet and blood glucose and lipids in non-insulin dependent diabetes (NIDDM). Nutr Res 15:843-857

28. Wheeler ML, Dunbar SA, Jaacks LM, Karmally W, Mayer-Davis EJ, Wylie-Rosett J et al (2012) Macronutrients, food groups, and eating patterns in the management of diabetes. A systematic review of the literature. Diabetes Care 35:434-445

29. Rivellese AA, Iovine C, Ciano O, Costagliola L, Galasso R, Riccardi G et al (2006) Nutrient determinants of postprandial triglyceride response in a population-based sample of type II diabetic patients. Eur J Clin Nutr 60:1168-1173

30. Chen L, Pei JH, Kuang J, Chen HM, Chen Z, Li ZW, Yang HZ (2015) Effect of lifestyle intervention in patients with type 2 diabetes: a meta-analysis. Metab Clin Exp 64:338-347 